\title{
Preface: Current research progress on mechanics of high speed rail
}

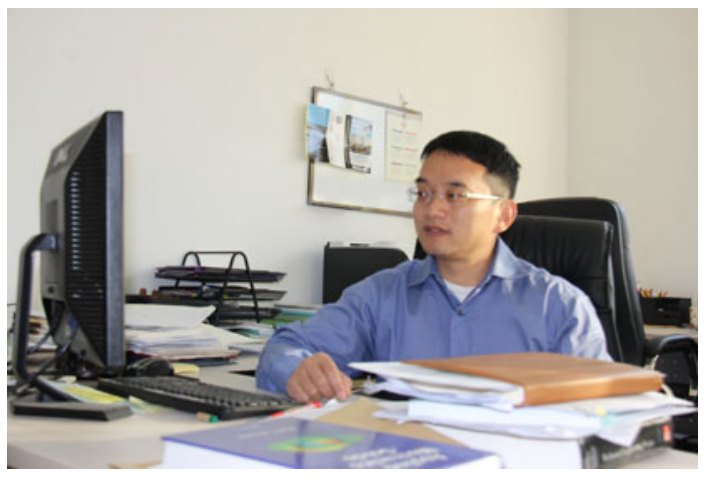

His research interests in solid mechanics are in the areas of constructing strength-structure relationship of novel materials, revealing behind deformation mechanisms, and developing predictive constitutive models to capture the mechanical deformation of those materials. He is also keen to promote the application of mechanics in sustainable development of infrastructures.

Professor Wei is the author of over 50 papers including publications in Nature, Nature Materials, Nature Communications, PNAS, Nano Letters, JMPS, and so on. In 2010 he was selected in the "Hundred Talent Program" from CAS, and received the 2013 Young Investigator Award from CSTAM. In 2014, he received support for "Distinguished Young Scholars" from the National Natural Science Foundation of China.

e-mail: yujie_wei@LNM.imech.ac.cn

The construction and operation of high-speed rail (HSR) grid within the past two decades in China, in terms of the scale, may be possibly comparable to any national-wide construction in the history of China, even the Great Wall. By counting railways with commercial train service at the speed of $200 \mathrm{~km} / \mathrm{h}$, China has the world's longest HSR network with over $19369.8 \mathrm{~km}$ of track in service today and this number is still increasing. The broad interests in HSR transportation originates from the currently global pressure to reduce $\mathrm{CO}_{2}$ emission (Fig. 1a), as addressed in the Climate Conference in Copenhagen in 2009 and up-to-date research. Unlike old fashioned trains with huge steam locomotive, high-speed trains are powered by electricity other than fossil energies with high $\mathrm{CO}_{2}$ emission. For most green energy sources including nuclear energy, solar energy, wind energy, and hydro-energy, their direct and efficient output is electricity. HSR hence supplies a desired solution for urgent green transportation development. While the unprecedented project is facing both praise and critics by different groups from distinct perspectives, researchers and engineers have to focus their attention on understanding the HSR system better to ensure the safety, to lower the cost of maintenance, to enhance comfort, and to make it a greener transportation.

By definition, mechanics is a subject to develop understanding among force, motion and deformation. Research on the motion of high speed trains and the safety of HSR systems hence belongs to the central task of mechanicians. Ever since James Watt, a Scottish mechanical engineer who invented the steam engine, mechanical engineers have been developing railways to be essential to the swift movement of goods and labour that is needed for modern society. Now it is of the top priority of Chinese engineers to understand well the motion of a high speed train, to ensure the world's longest HSR network in good operation. A high-speed train (HST) in operation interacts with external structures/environments from three major boundaries: 

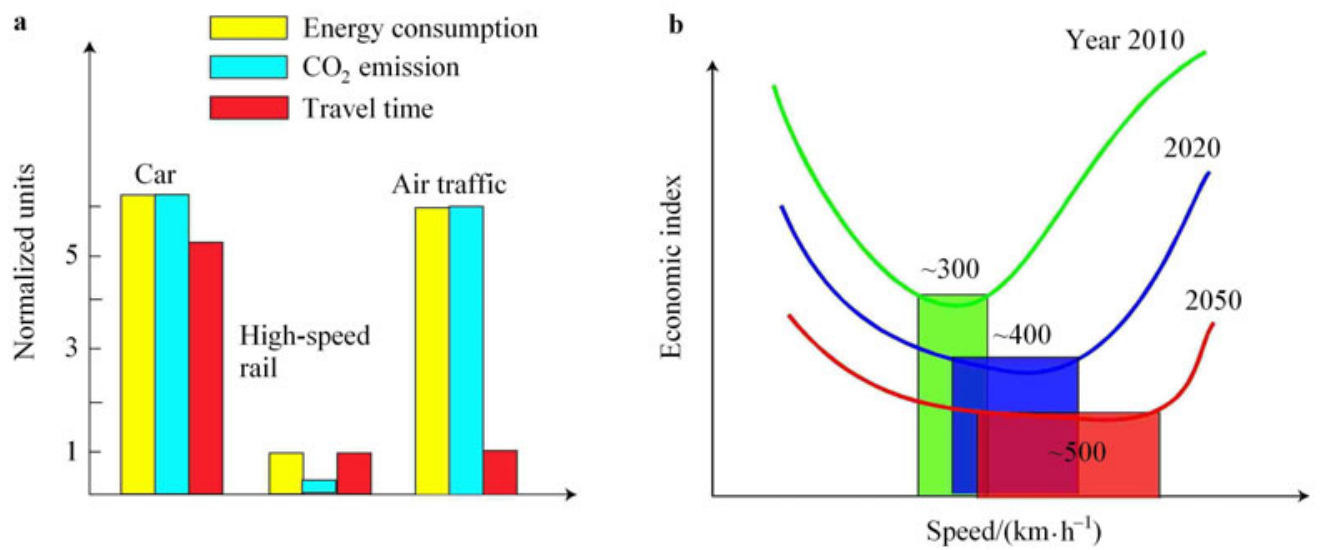

Fig. 1 Competitiveness of high-speed rails. a Comparison of energy and travelling efficiency for several transportations for 1000km journey (based on travelling between Wuhan-Guangzhou in China); b Illustration for the optimal speed (shadowed regions) with low economic index - a factor depends on HSR speed, population, culture, et al., and stands for more economic if it is smaller. With the advances of technologies and sciences, HSRs could be faster, cheaper, and greener.

(1) The train obtains electric energy from catenarypantograph systems;

(2) Wheels receiving propulsions via the frictional interaction between wheels and rails, carry the vehicles moving forward;

(3) While the vehicle running at high speed, the body faces aerodynamic resistance, which is subjected to change with environments.

Systematic understanding about the dynamics of catenary-pantograph systems, the interaction of wheel-rail systems, and the aerodynamics of the train body are hence crucial for the safe operation of HSR and further development of HSR technology. In this special issue, we invited several distinguished groups in the field to report their recent research progress on mechanics related problems in HSR. Considering the complex of the HSR system and the number of people involved in the field, this special issue by no means would be a comprehensive one. We will cover more research progress on the mechanics of HSR in later issues.

We introduce the works in this issue based on the bottom-up logic, by introducing work on the rail-wheel interaction, wheel-bogie system, wheel-carbody system, dynamics and related issues of carbody, in turn. The wheel-rail interaction is critical for rolling and lateral motion, and the latter accounts for derailment, one type of possibility for accident. The research by Jin et al. (P860) is to develop understanding about how to identify causes responsible for derailment of high speed trains operating in severe environments. To fulfil this goal, the authors developed a three-dimensional dynamic model of a high-speed train coupled with a ballast track for dynamic derailment analysis. Their results suggest that the train and track modelling options have a significant effect on the dynamic derailment analysis. The inter-vehicle impacts and the track flexibility and nonlinearity should be considered in the dynamic derailment simulations.

On the top of wheels are bogies. They serve a number of purposes such as support of the rail vehicle body, steer the direction and maintain stability on both straight and curved track, ensure ride comfort by absorbing vibration and minimizing the impact of centrifugal forces when the train runs on curves at high speed, as well as minimizing generation of track irregularities and rail abrasion. Any failure in bogies may lead to catastrophic accident of a HST. Hence the reliability of the bogie is of top importance in the train system. The research conducted by Zou et al. (P901) is to abstract the load spectra on bogie frame structures of high-speed trains. The authors designed a force-measuring frame to enable load decoupling. In this manner, quasi-static load-time histories, together with online tests and decoupling analysis, are obtained for the intermediate range of the Beijing-Shanghai Dedicated Passenger Line. This information are hence used as input of a damage damage consistency criterion to assess the reliability of bogie frames.

With speed increasing, the wheel-rail interaction becomes severer; dynamics of the wheelset will be transimitted to carbody via bogies. It becomes necessary to investigate the vibration characteristics and the dynamic frequency transmission from the wheel-rail interface to the carbody. 
Ren et al. (P876) established an elastic high-speed vehicle dynamics model, with components like the carbody, bogieframes and wheelsets being taken into account. The results show that the spectrum power density of the vehicle decreases from the wheelset to the carbody and the acceleration transmission ratio is approximately from $1 \%$ to $10 \%$ for each suspension system. A field test data of the high speed train are also presented to validate the simulation results. It shows that the simulation results are coincident with the field test data.

The carbody, while being influenced by the dynamics of wheelsets, receives steady aerodynamic loads. Those loads not only dominate the resistance to motion, but also play an important role on hunting stability of high-speed trains. The research by Zeng et al. (P889) is to explain the change of hunting behavior due to actions of aerodynamic loads, and revealed that the aerodynamic loads can change the position of vehicle system (consequently the contact relations), the wheel/rail normal contact forces, the gravitational restoring forces/moments and the creep forces/moments. They also coded those factors in a mathematical model for hunting stability to obtain the safety bound of speeds.

In addition to mechanical problems at the structural level, the safety of typical component in HST has also been investigated. For examples, Li et al. (P849) investigated the welding distortion for double floor structure of high speed train. The welding heat source models and the plastic tension zone sizes of a typical weld joint involved in the double floor structure of high speed train under different welding parameters were calculated by a thermal-elastic-plastic FEM analysis based on SYSWELD code. The authors reported that beyond the welding sequence, the clamping configuration and reverse deformation all have a significant influence on the welding distortion of floor structure.

Li et al. (P884), on the other hand, explored ways to harvest kinetic energy of rail vibration by using piezoelectric circular diaphragm. In the frequency spectra of $110 \mathrm{~Hz}$ to $260 \mathrm{~Hz}$, the authors observed three output power peaks. Their work demonstrated that a piezoelectric circular diaphragm array can increase significantly the output of energy compared with the use of a single plate.

With the opening of the backbone HSR network composed of 4-longitudinal and 4-latitudinal lines and its operation to service $90 \%$ of her population in China, it is always not sufficient to emphasize the significance of research and technological innovation to ensure the safety of the system. Like most huge engineering projects, high-speed rails involve the knowledge of mechanics, material, control, communication, electronics, manufacturing, energy, and so on. Breakthroughs in strong yet light materials, corrosion and wear resistant materials for brake system, wheel, rail and other structures in frequent contact with others, reliable theories on high circle fatigue and fracture, and energy research are desired. While HSRs are known to be energy efficient (Fig. 1b), high capacity batteries will find their usage in HSRs for transitional regions as they pass metropolitan area or special tunnels at relatively lower speed, in order to circumvent the difficulty of building electric grid in those regimes. With the need of low carbon and energy efficient transportation, we expect that HSRs would be a primary choice for travels with distances in the range of $300 \mathrm{~km}$ to $1500 \mathrm{~km}$ in Asia and Europe in the near future. 